\title{
Magnetic Properties of Substituted Poly(thiophene)s in Their Neutral State.
}

Steven Vandeleene ${ }^{a, d}$, Mihaela Jivanescu ${ }^{b, d}$, André Stesmans ${ }^{b, d}$, Jo Cuppens $^{c, d}$, Margriet J. Van Bael ${ }^{c, d}$, Hitoshi Yamada ${ }^{e}$, Norio Sato ${ }^{f}$, Thierry Verbiest ${ }^{a, d}$, and Guy Koeckelberghs ${ }^{a, d *}$

${ }^{\text {a }}$ Laboratory of Molecular Electronics and Photonics, Katholieke Universiteit Leuven, Celestijnenlaan 200F, B-3001 Heverlee, Belgium

${ }^{\mathrm{b}}$ Semiconductor Physics Laboratory, Katholieke Universiteit Leuven, Celestijnenlaan 200D, B-3001 Leuven, Belgium

${ }^{\mathrm{c}}$ Laboratory of Solid State Physics and Magnetism, Katholieke Universiteit Leuven, Celestijnenlaan 200D, B-3001 Leuven, Belgium

${ }^{\mathrm{d}}$ INPAC -Institute for Nanoscale Physics and Chemistry, University of Leuven, Belgium

e Partner Robot Division, Toyota Motor Corporation, 543, Kirigahora, Nishihirose-cho, Toyota, Aichi, 470-0309, Japan

${ }^{\mathrm{f}}$ Technical Centre of Research \& Development, Toyota Motor Europe, Hoge Wei 33, B-1930 Zaventem, Belgium

guy.koeckelberghs@chem.kuleuven.be

RECEIVED DATE (to be automatically inserted after your manuscript is accepted if required according to the journal that you are submitting your paper to) 
Abstract. The magnetic behavior of undoped (neutral), substituted poly(thiophene)s is reported. In particular, the influence of the nature of the substituent (alkyl, alkoxy, thioalkyl), the substitution pattern (head-to-tail (HT) versus head-to-head-tail-to-tail (HH-TT)), and the regioregularity on the magnetic properties has been investigated. ESR spectroscopy reveals that the nature of the substituent determines the spin density, while the line width and asymmetry of the ESR signals are mainly governed by the substitution pattern and regioregularity. The spins give rise to a paramagnetic behavior. SQUID magnetometry reveals the presence of superparamagnetic order at room temperature, while ferromagnetism is observed at $5 \mathrm{~K}$. The magnetic behavior observed by SQUID magnetometry does not (solely) originate from the ESR-active spin system. Its strength does therefore not depend on the ESR spin density, but seems to be governed by the supramolecular structure.

Keywords. Conjugated polymers, magnetism, ESR, poly(thiophene)s.

\section{Introduction}

Conjugated polymers show a broad variety of optical, electrical and electronic properties which are widely exploited in a broad range of applications ${ }^{1}$. Their magnetic behavior, on the other hand, is far less studied and usually only a diamagnetic contribution is expected. In general magnetism in molecules arises from the intrinsic spin of unpaired electrons. A mutual magnetic interaction between these spins aligns them parallel or antiparallel, affording magnetic order. To achieve ferromagnetism in a (organic) molecule the presence of unpaired electrons with the same spin orientation in the same molecule is required. However, designing a material which exhibits magnetism on a macroscopic scale requires the cooperative orientation of spins on different molecules as well. Since organic (conjugated) polymers usually lack the presence of unpaired electrons, no magnetic properties are expected. Therefore, efforts to introduce magnetism in organic (polymer) materials have been focussing on the development of (conjugated) materials in which the spins are deliberately introduced. A first approach consists in the incorporation of organic, spin-carrying moieties, such as radicals ${ }^{2}$ or carbenes ${ }^{3}$ in the polymer chains, either as main chain or as side-chain, resulting in organic magnets. In this approach, the use of 
conjugated polymers or oligomers as linking units between the subsequent spins offers an important additional advantage, i.e. the strong through-bond coupling, which allows alignment of the spins within the same (polymer) molecule. If the molecules are designed in such a way that they satisfy a conjugated but non-Kekulé and nondisjoint connectivity among the nonbonding molecular orbitals of the unpaired electrons, the spins within one conjugated polymer chain couple ferromagnetically, which results in a 'high-spin' (polymer) molecule ${ }^{4}$. Alternatively, unpaired spins can also be introduced in conjugated polymers by chemical or electrochemical oxidation (reduction), creating polarons. Transpoly(acetylene), finally, is a conjugated polymer in which spins can be present in the neutral state without introducing a spin-carrying group. The spins ('solitons') ${ }^{5}$ are an intrinsic feature of this class of polymers, but are restricted to non-aromatic conjugated polymers.

A few conjugated polymers have been reported to show magnetic properties in particular conditions. Examples of such materials constitute doped poly(aniline)s ${ }^{6}$ and poly(pyrrole)s ${ }^{7}$, doped regio-irregular (and therefore poorly organized) poly(3-alkylthiophene)s ${ }^{8}$ and doped and/or derivatized poly(acetylene) $\mathrm{s}^{9}$.

In this manuscript, we report on the magnetic spin densities of substituted poly(thiophene)s in their neutral state and in which no spin-carrying groups are introduced (Chart 1). It is investigated whether the polymers show a (super)paramagnetic or even ferromagnetic behavior. The magnetic properties of the polymers are correlated with their (supra)molecular structure.

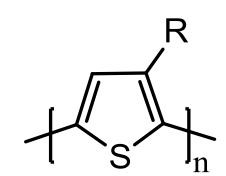

HT-P3AT

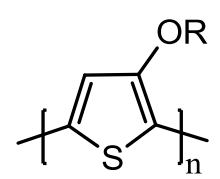

HT-P3AOT

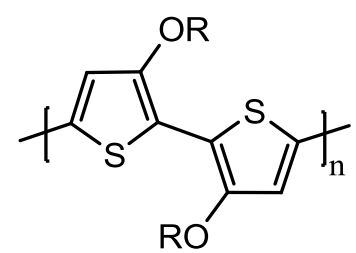

HH-TT-P3AOT

$\overline{\mathrm{M}}_{\mathrm{n}}=9.7 \mathrm{~kg} / \mathrm{mol}$

$\overline{\mathrm{X}}_{\mathrm{n}}=20.3$

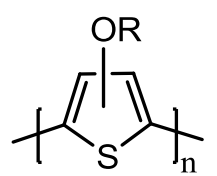

GRIM-P3AOT

$\overline{\mathrm{M}}_{\mathrm{n}}=2.3 \mathrm{~kg} / \mathrm{mol}$

$\overline{\mathrm{X}}_{\mathrm{n}}=9.6$

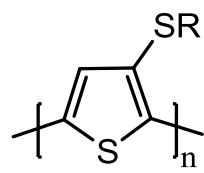

HT-P3ATT

$\overline{\mathrm{M}}_{\mathrm{n}}=3.6 \mathrm{~g} / \mathrm{mol}$

$\overline{\mathrm{X}}_{\mathrm{n}}=14.1$<smiles>[R]C#CCC[C@H](C)CCCC(C)C</smiles>

Chart 1. Structure, number-averaged molar mass $\left(\bar{M}_{n}\right)$ and degree of polymerization $\left(\bar{X}_{n}\right)$ of the poly(thiophene)s studied. 


\section{Materials and Methods}

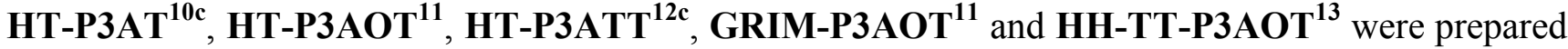

and purified as described elsewhere. The number-averaged molar mass $\left(\overline{\mathrm{M}}_{n}\right)$ of the polymers was measured by gel permeation chromatography (GPC) using a Shimadzu 10A apparatus with a tunable absorbance detector and a differential refractometer in tetrahydrofuran (THF) as eluent toward polystyrene standards.

Prior to the ESR and SQUID experiments, the polymers were reduced by subsequent washing the polymer powders with an alcoholic hydrazine solution, methanol and finally dried under an argon flow. The powders were weighted and stored under inert (argon) atmosphere.

Conventional continuous slow-passage X-band $(\sim 9.2 \mathrm{GHz})$ ESR measurements were carried out at room temperature using a Jeol FA100 spectrometer, where low microwave power $\left(\mathrm{P}_{\mu} \leq 1 \mathrm{~mW}\right)$ first derivative-absorption $\mathrm{dP}_{\mu} / \mathrm{dB}$ spectra were recorded through applying sinusoidal modulation $(\sim 100 \mathrm{kHz}$; amplitude $\mathrm{B}_{\mathrm{m}} \sim 0.35 \mathrm{G}$ ) of the externally applied magnetic field $\mathbf{B}$. Some observations were made in a mode-built K-band ( $20.6 \mathrm{GHz})$ setup, as described elsewhere ${ }^{14}$. A co-mounted calibrated $\mathrm{MgO}: \mathrm{Mn}^{2+}$ reference sample was used for absolute g factor and spin density calibration, with the latter performed through orthodox double numerical integration of the detected $\mathrm{dP}_{\mu} / \mathrm{dB}$ spectra. At $\mathrm{X}$ band, the $\mathrm{g}$ values of the $3^{\text {rd }}$ and $4^{\text {th }}$ lines of the $\mathrm{Mn}^{2+}\left({ }^{55} \mathrm{Mn} ; 100 \%\right.$ natural abundance; nuclear spin $\left.\mathrm{I}=5 / 2\right)$ sextet were calibrated as $2.0338 \pm 0.0001$ and $1.9807 \pm 0.0001$, respectively. The attained absolute and relative accuracy is estimated at $\sim 20 \%$ and $\sim 5 \%$, respectively. Signal averaging (typically $\sim 50$ scans) was routinely applied to enhance spectral quality.

SQUID-based magnetometry is carried out in a MPMS-XL magnetometer (Quantum Design). The powders are weighted and a typical amount of $15 \mathrm{mg}$ is fixed between small pieces of pure cotton wool inside the non-magnetic plastic sample tube. No capsules are used to hold the powder in order to avoid any magnetic contributions from the capsules interfering with the measured signal. The magnetic signal from the plastic sample tube with cotton wool (without the powder sample) is measured separately and shows a small diamagnetic contribution which is linear in applied field and can therefore be 
distinguished from non-linear contributions from the powders. Magnetization was measured at different fixed temperatures as a function of magnetic field in RSO (reciprocating sample option) operation mode.

\section{Results and Discussion}

\section{Materials}

The materials studied constitute poly(thiophene)s bearing alkyl (P3ATs), alkoxy (P3AOTs) or thioalkyl (P3ATTs) substituents (see Chart 1). All polymers were prepared via established methods (('modified') McCullough ${ }^{10 \mathrm{a}}$ and GRIM $^{10 \mathrm{~b}}$ methods and using Stille couplings), which produce essentially defect-free conjugated polymers. ${ }^{15}$ It is worthwhile to mention that while the polymerization of all other polymers is based on a Ni-catalyzed Kumada reaction, HH-TT-P3AOT is prepared using a Pd-catalyzed Stille cross coupling reaction, in which no Ni-species are employed. After polymerization, all polymers were thoroughly washed with acetone and $n$-hexane in order to remove any impurities, especially those arising from the catalyst, as they might influence the magnetic measurements (ESR and SQUID).

All polymers were substituted with the same branched chiral group, since HT-P3AOTs and especially HT-P3ATTs equipped with linear (achiral) groups show a very poor solubility ${ }^{12}$, which complicates a proper purification. The use of a branched (chiral) substituent circumvents this shortcoming and allows a thorough purification.

Three parameters in the (supra)molecular structure of the polymers were varied. First, the nature of the substituent (HT-P3AT, HT-P3ATT and HT-P3AOT), as alkoxy- and thioalkyl-groups can stabilize spins. Second, the substitution pattern (HT-P3AOT and HH-TT-P3AOT) was changed: while the thiophene monomers in HT-P3AOT are arranged in a head-to-tail fashion, HH-TT-P3AOT is composed of alternating head-to-head and tail-to-tail couplings. A final parameter is the regioregularity: HT-P3AOT and HH-TT-P3AOT are both regioregular (albeit with a different substitution pattern); in contrast, GRIM-P3AOT contains all possible couplings randomly distributed along the polymer backbone and is therefore regio-irregular. Apart from these molecular parameters, it is also interesting to 
mention that HT-P3AT, HT-P3ATT and HH-TT-P3AOT show clear melting peaks (as observed by different scanning calorimetry) and are therefore semi-crystalline, while HT-P3AOT and GRIMP3AOT are not.

\section{ESR spectroscopy}

First, the magnetic behavior of the polymers was investigated by ESR spectroscopy. Generally, for all samples studied at room temperature, only one, rather narrow signal is observed, of which some ESR signal parameters and inferred quantities are summarized in Table 1 . These imply the spin density, zero crossing $g$ value $\left(g_{c}\right)$, peak-to-peak line width $\left(\Delta B_{p p}\right)$ and signal symmetry parameter $A / B$ (ratio of the low field to the high field peak heights of the measured absorption-derivative spectra). In Figure 1, a representative X-band ESR spectrum observed at $300 \mathrm{~K}$ of HT-P3ATT is displayed, showing the presence of a rather narrow signal $\left(\Delta B_{p p}=5.3 \mathrm{G}\right)$ at $\mathrm{g}_{\mathrm{c}}=2.0044$. The general signal shape and specific features point to a powder pattern line shape, as might be expected from the studied polymer powder samples. This also explains the observed signal asymmetry; the alternative possibility - (two) overlapping signals of slightly differing g value and line width - can be ruled out from additional ESR observations at higher observational frequency (K-band; $20.6 \mathrm{GHz}$ ). As illustrated by the dotted curve, the signal can be adequately fitted to a powder pattern shape of a center with principal $g$ values $\mathrm{g}_{1}=2.00562, \mathrm{~g}_{2}=2.00458, \mathrm{~g}_{3}=2.00242$, i.e., of nearly axial symmetry. While perhaps less obvious for HT-P3AT, the powder pattern signature is observed in all samples, most expressively for HT-P3ATT. 


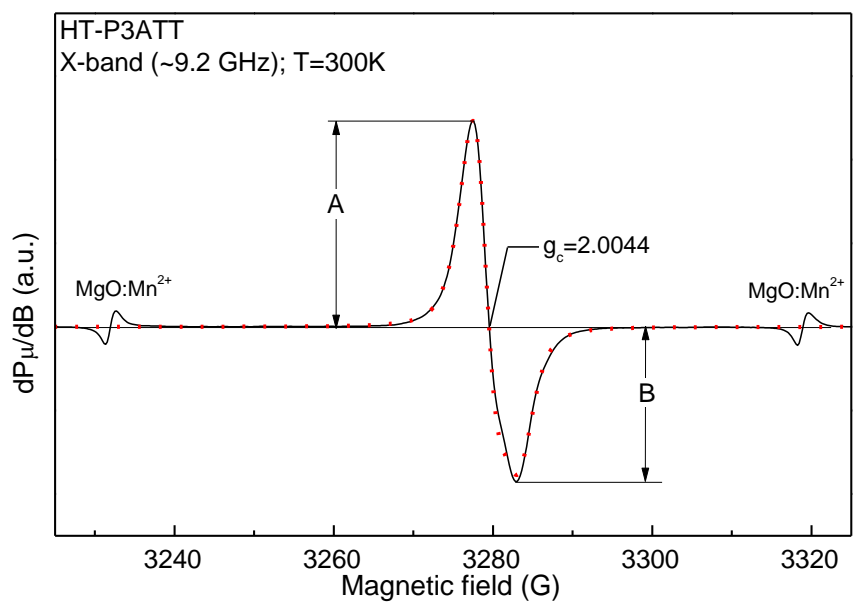

Figure 1. X-band first derivative-absorption ESR spectrum of HT-P3ATT, using $B_{m} \sim 0.35$ G and $P_{\mu}=1$ $\mathrm{mW}$, showing a main signal at $\mathrm{g}_{\mathrm{c}}=2.0044$. The outer weak doublet signals stem from a co-mounted MgO: $\mathrm{Mn}^{2+}$ marker signal. The dotted curve represents a powder pattern simulation obtained using the principal $g$ matrix values $\mathrm{g}_{1}=2.00562, \mathrm{~g}_{2}=2.00458, \mathrm{~g}_{3}=2.00242$, a convolution Voigt line shape function of Lorentzian-to-Gaussian fraction 1.96, and skewed Gaussian distributions of spread (in magnetic field units) $\sigma g=4.5 \mathrm{G}, 3 \mathrm{G}$, and $2.9 \mathrm{G}$ on the principal $\mathrm{g}$ values $\mathrm{g}_{1}, \mathrm{~g}_{2}$, and $\mathrm{g}_{3}$, respectively.

A rather noteworthy ESR feature is the sharpness of the observed signal, which points to a distinct delocalization of the unpaired electrons, in accordance with the g matrix properties. Indeed, while the zero crossing $\mathrm{g}$ factor of all polymers shows a (small) deviation from the free electron value $\left(\mathrm{g}_{\mathrm{e}}=\right.$ 2.00232), the simulations show that the axial axis principal $g$ value $\left(\mathrm{g}_{/ /}\right)$remains close to $\mathrm{g}_{\mathrm{e}}$. The $\mathrm{g}$ shift $\Delta \mathrm{g} \equiv \mathrm{g}_{\mathrm{c}}-\mathrm{g}_{\mathrm{e}}$ therefore primarily comes from the shift in $\mathrm{g}_{\perp}$. This $\mathrm{g}$ shift $\Delta \mathrm{g}$, caused by $\mathrm{g}$ factor anisotropy, is clearly dependent on the nature of the substituent $\left(\mathrm{g}_{\mathrm{P} 3 \mathrm{AT}}=2.0036, \mathrm{~g}_{\mathrm{P} 3 \mathrm{AOTs}}=2.0027, \mathrm{~g}_{\mathrm{P} 3 \mathrm{ATT}}=2.0043\right)$, but rather insensitive to the substitution pattern. The influence of the substituent demonstrates that the spins are not only delocalized over the polymer backbone, but on the heterogeneous atoms of the substituent as well. These findings are in accordance with the fact that also other electronic properties of the poly(thiophene)s, such as $\lambda_{\max }$, are greatly affected by the nature of the substituent, but far less by the substitution pattern. 
Table 1. ESR signal parameters and quantities, the number of Bohr magnetons calculated from the saturation magnetization measured by SQUID and the coercivity. All measurements were done at room temperature and in the neutral state, unless otherwise stated.

\begin{tabular}{|c|c|c|c|c|c|c|c|c|}
\hline & $\begin{array}{c}\text { spin densit } \\
\text { neutral }\end{array}$ & $\begin{array}{l}(\text { spins } / g)^{a} \\
\text { oxidized }\end{array}$ & $\begin{array}{l}\text { spins/ } \\
\text { unit }^{\text {a }}\end{array}$ & $\mathrm{g}$ factor $^{\mathrm{a}}$ & $\begin{array}{c}\Delta \mathrm{B}_{\mathrm{pp}}(\mathrm{G}) \\
( \pm 0.1 \mathrm{G})^{\mathrm{a}}\end{array}$ & $\mathrm{A} / \mathrm{B}^{\mathrm{a}, \mathrm{b}}$ & $\begin{array}{c}\text { Bohr } \\
\text { magnetons } / g^{c}\end{array}$ & $\begin{array}{l}\text { Coercivity } \\
(\mathrm{Oe})^{\mathrm{d}}\end{array}$ \\
\hline HT-P3AT & $1.8 \mathrm{E}+16$ & $7.3 \mathrm{E}+16$ & $6.7 \mathrm{E}-06$ & 2.0036 & 5.3 & 0.91 & $1.7 \mathrm{E}+17$ & 254 \\
\hline HT-P3AOT & $2.7 \mathrm{E}+17$ & $8.1 \mathrm{E}+17$ & $1.0 \mathrm{E}-04$ & 2.0027 & 3.2 & 0.80 & $1.6 \mathrm{E}+18$ & 180 \\
\hline HH-TT-P3AOT & $9.5 \mathrm{E}+17$ & $9.5 \mathrm{E}+17$ & $3.6 \mathrm{E}-04$ & 2.0026 & 4.0 & 0.73 & $1.2 \mathrm{E}+17$ & 231 \\
\hline GRIM-P3AOT & $6.4 \mathrm{E}+16$ & $6.4 \mathrm{E}+16$ & $2.4 \mathrm{E}-05$ & 2.0029 & 4.3 & 0.72 & $2.6 \mathrm{E}+18$ & 55 \\
\hline HT-P3ATT & $3.8 \mathrm{E}+16$ & $5.6 \mathrm{E}+16$ & $1.5 \mathrm{E}-05$ & 2.0043 & 5.3 & 1.34 & $1.6 \mathrm{E}+18$ & 755 \\
\hline
\end{tabular}

${ }^{\text {a }}$ determined by ESR

${ }^{\mathrm{b}} \mathrm{A}$ and $\mathrm{B}$ are defined in Figure 1.

${ }^{c}$ determined by SQUID calculated from the magnetic moment at saturation by assuming that the magnetic response originates from the spinmagnetic moment of unpaired electrons.

${ }^{\mathrm{d}}$ determined by SQUID at $5 \mathrm{~K}$.

As demonstrated, the apparent ESR signal asymmetry, reflected in the deviation of the A/B ratio from unity, results from the presence of (weak) g matrix anisotropy in combination with the powder pattern aspect. Apart from the substituent effect, Kanemoto et al. suggested this phenomenon to originate from the transfer of spins among several crystalline grains ${ }^{16}$. However, this requires the polymers to be semicrystalline, which is the case for HT-P3AT, HH-TT-P3AOT and HT-P3ATT, but not for HT-P3AOT and GRIM-P3AOT. On the other hand, it should be mentioned that, although the latter polymers are not semi-crystalline, some interchain interactions exist, as is shown by their magnetic properties (see further). Possibly, their grain size is too small to be clearly visualized by DSC, but sufficiently large for the magnetic ordering and the ESR signal asymmetry.

The line widths for the measured polymers correspond to the line widths reported for poly(thiophene)s ${ }^{17}$. Apart from the Elliott broadening ${ }^{17 b-18}$, caused by the presence of atoms with higher molar weights such as sulphur in the poly(thiophene) backbone, the line widths seem to be influenced by the nature of the substituent and by the substitution pattern. HT-P3AOT, which is exclusively 
composed of HT-couplings, shows the sharpest line. HH-TT-P3AOT, consisting of HH- and TTcouplings, shows an intermediate line width and GRIM-P3AOT, in which all possible couplings are present and randomly distributed along the polymer backbone, has the broadest line. The comparison of HT-P3AT, HT-P3AOT and HT-P3ATT reveals that the presence of oxygen atoms narrows the line widths, which in view of the revealed powder pattern effect, evidently arises from a reduction in $g$ matrix anisotropy. It needs to be mentioned that since exposure to ambient air was carefully avoided, the presence of ambient oxygen, and more in particular the interaction with ${ }^{3} \mathrm{O}$, cannot account for occasional broadening of the lines ${ }^{19}$.

The spin densities of the polymers are strongly dependent on the nature of the substituent: the alkoxysubstituted polymers show the highest spin densities and HT-P3AT has the lowest spin density. On the other hand, the substitution pattern seems to have no significant effect on the spin density. The influence of the nature of the substituent can be attributed to the stabilization of unpaired electrons by heteroatoms, especially oxygen. In a broader view, it might be correlated with the presence of strongly electron-withdrawing and/or -donating groups. For instance, Yamamoto et al. reported the presence of spins in a neutral nitro-substituted conjugated polymer ${ }^{20}$. It should be mentioned that the reduction with hydrazine and careful manipulation in the absence of air excludes the possibility that the observed spin densities originate from the presence of oxygen or any other oxidant, which can result in polarons by direct or light-induced oxidation, (as has been reported for poly(3-hexylthiophene) $)^{21}$. Second, the large number of spins can neither be due to radical defects ${ }^{15}$ or trapped polarons ${ }^{22}$, originating from structural defects, since the polymerization methods used are known to produce essentially defect-free conjugated polymers. For instance, it has been shown that the Ni-catalyzed polymerization which has been employed for the synthesis of all polymers except HH-TT-P3AOT, results in HT-P3ATs in which the sole 'defect' is a TT-coupling at the beginning of each polymer chain. Finally, also Ni-contamination cannot be the origin of the spins, since HH-TT-P3AOT, which is polymerized in the absence of any Nisource, shows comparable spin densities. Moreover, no typical ESR signature of Ni species could be observed. Therefore, it is concluded that the observed spin densities are not due to any 'external' source, 
but are an intrinsic feature of (substituted) poly(thiophene)s. This is also supported by the fact that we found similar spin densities in other HT-P3ATs. Indeed, the spin density appeared to be invariable if another batch of the same polymer was measured, or if the side-chain (n-dodecyl versus 3,7dimethyloctyl) was varied, or if polymers prepared via a slightly different polymerization methodology (McCullough versus GRIM) were evaluated.

Although the polymers show spin densities which are significantly larger than their monomeric counterparts, this still corresponds with less than 1 spin per 3000 repeating units. Given the degree of polymerization of the polymers $\left(\overline{\mathrm{X}}_{\mathrm{n}}\right.$, Chart 1$)$, this implies that each polymer chain contains maximum one spin (assuming a homogeneous distribution) and consequently, the spins cannot couple (ferromagnetically or anti-ferromagnetically) through the $\pi$-system of the polymer. As a consequence, only $\mathrm{S}=1 / 2$ spins can be present, which is also observed.

Next, the time evolution of the spin density was recorded after bringing the powders into contact with ambient air. The process was repeated until no further changes in the spin densities were observed, which took about 10 days. It is clear that the presence of ambient oxygen in general gradually increases the spin density, which can be attributed to a slight oxidation or the formation of a light-induced charge transfer complex with $\mathrm{O}_{2}^{21}$.

Finally, the temperature dependency of the magnetic susceptibility was measured, which was done on HT-P3ATT (Figure 2). The polymer shows a typical paramagnetic Curie-Weiss behavior with a Curietemperature of $-1 \pm 3 \mathrm{~K}$. These results are fully in agreement with the previous experiments, demonstrating the presence of isolated, non-interacting spins. 


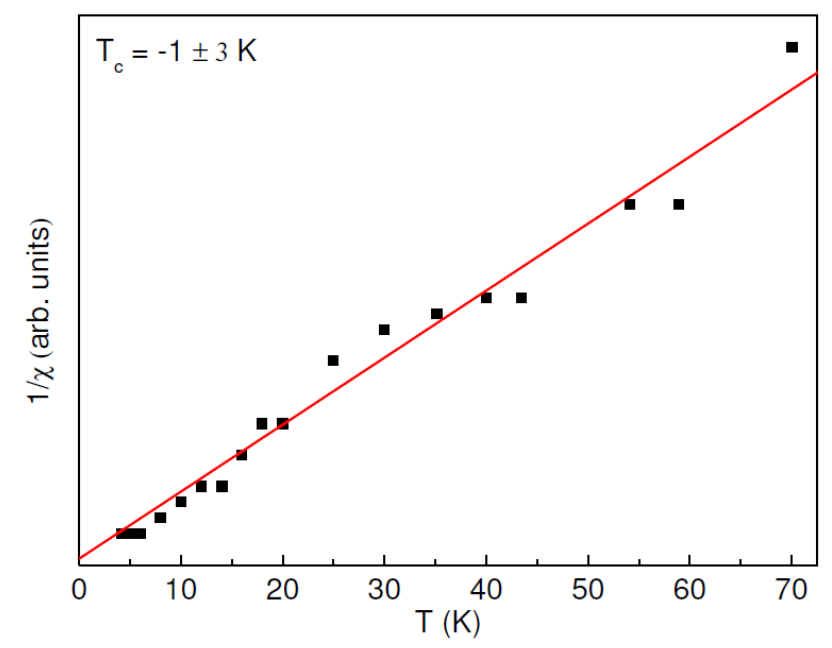

Figure 2. Temperature-dependence of the magnetic susceptibility measured by ESR spectroscopy of

\section{HT-P3ATT.}

\section{SQUID magnetometry}

The magnetic properties of the samples were measured by SQUID magnetometry on the same powders as were used for the ESR experiments. A typical magnetization (M) versus applied magnetic field $(\mathrm{H})$ curve is shown in Figure 3a. All samples show a clear diamagnetic contribution, on which a (super)paramagnetic or ferromagnetic contribution is superimposed. In Figures $3 \mathrm{~b}-\mathrm{g}$ the $\mathrm{M} / \mathrm{H}$ magnetization curves of all polymers at 5 and $300 \mathrm{~K}$ are displayed from which the linear diamagnetic contribution is subtracted. From these curves, it is clear that at higher temperature $(300 \mathrm{~K})$ all polymers show superparamagnetic behavior characterized by a large magnetic susceptibility ( $\mathrm{dM} / \mathrm{dH}$ at zero field) and a, albeit weak, ferromagnetic contribution at low temperature (5 K). Only for HT-P3AOT and GRIM-P3AOT, the ferromagnetic behavior is less pronounced.
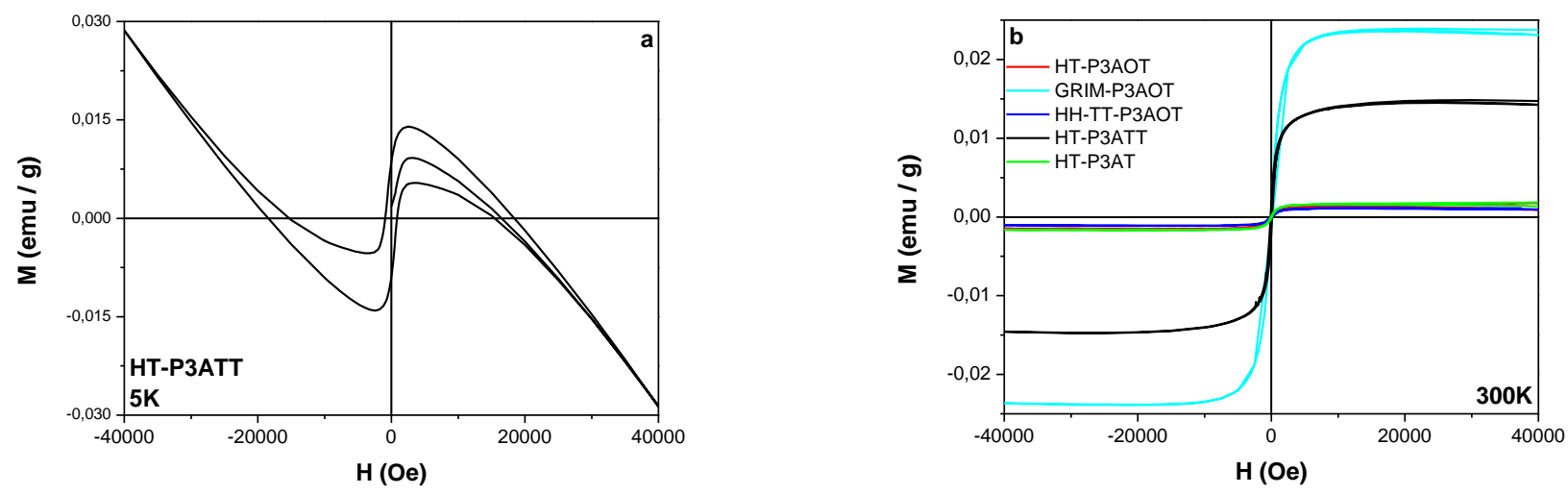

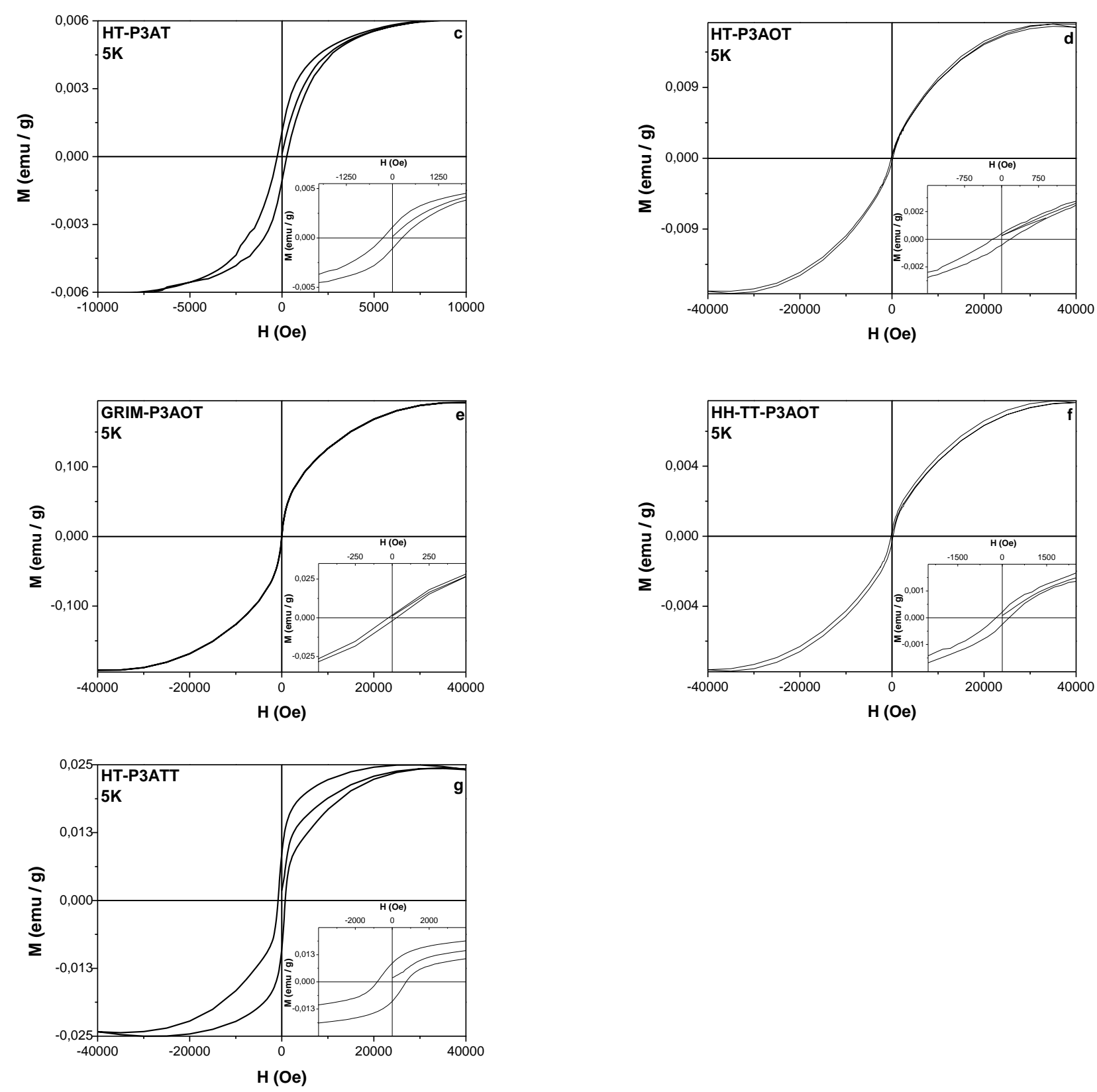

Figure 3. (a) A representative magnetic hysteresis loop of HT-P3ATT at $300 \mathrm{~K}$, (b) the magnetic hysteresis loops of all polymers at $300 \mathrm{~K}$ after subtraction of the diamagnetic contribution and the individual hysteresis loops of at $5 \mathrm{~K}$ after subtraction of the diamagnetic contribution of (c) HT-P3AT, (d) HT-P3AOT, (e) GRIM-P3AOT, (f) HH-TT-P3AOT and (g) HT-P3ATT.

The diamagnetic response can be attributed to the large number of paired spins. The superparamagnetic behavior which evolves to ferromagnetism at low temperatures, however, clearly contrasts with the results of the ESR experiments, which revealed a typical Curie-Weiss paramagnetic 
behavior. The different outcome of the ESR and SQUID experiments is further expressed if the number of Bohr magnetons, calculated from the observed saturation magnetization at $300 \mathrm{~K}$ by assuming that the magnetic response originates from the spinmagnetic moment of unpaired electrons, is compared with the spin density measured by ESR spectroscopy (Table 1). This shows that, except for HH-TT-P3AOT, the number of Bohr magnetons, exceeds the number of spins by about 1 or 2 order of magnitude. Both observations suggest that the superpara/ferromagnetic behavior, observed by SQUID, does not (solely) originate from the spin system sensed by ESR, i.e. the electron spins. The origin and nature of the magnetic moments remains, however, unclear (except that they are not the electron spins), but again, it should be emphasized that the ferromagnetism is not due to contaminants, such as Ni, since HH-TTP3AOT, which is polymerized in the absence of any Ni-source, shows a similar magnetic behavior. Moreover, no traces of $\mathrm{Ni}$ contaminants were observed by ESR and AAS analysis of HT-P3ATT demonstrates that the $\mathrm{Ni}$ concentration is below $50 \mathrm{ppb}$.

Although the shape of the magnetization curves is similar for all polymers, the magnitude of their magnetic response differs significantly. The magnitude of the superparamagnetism can be evaluated by the total magnetic moment at saturation, while the ferromagnetism is characterized by the coercive field of the hysteresis curve. If those parameters are plotted as a function of the ESR spin density (Figure 4), it becomes clear that no correlation exists between the ESR spin density and the magnetic properties determined by SQUID magnetometry, which again demonstrates that the SQUID magnetic properties of the polymers are not governed by the ESR spin density.

All these observations can be correlated with the fact that poly(methacrylate)s substituted with nitroxide radicals do not show significant ferro- (or antiferro-) magnetism, despite their high spin density $^{23}$. The absence of such magnetic behavior demonstrates that the presence of ESR-active spins does not suffice for bulk ferromagnetism to be present. Indeed, applied on the poly(thiophene)s studied, the observed spin densities (assuming a homogeneous distribution) correspond to an average distance between the spins of $\sim 20 \mathrm{~nm}$, which is too large to allow direct exchange interactions. This explains the absence of significant ferromagnetism in radical-functionalized poly(methacrylate)s, but is in line with 
the paramagnetism observed by ESR spectroscopy. It also shows that the magnetic moments in the poly(thiophene)s, whatever their origin might be, must interact via another mechanism, resulting in the superpara- and ferromagnetism. In this respect the studied poly(thiophene)s differ from the radicalfunctionalized poly(methacrylate)s in the presence of the $\pi$-conjugated system. Spins on different, stacked polymer chains can interact through the $\pi$-system. In this way, the supramolecular structure of the polymer can determine the magnetic behavior, as it influences the $\pi$-interactions between the stacked polymer chains. The role of semi-crystallinity in this respect could be ambiguous: on the one hand, the interactions within the crystalline domains are optimal, but, on the other hand, $\pi$-stacking is often seriously impeded on the boundaries of the crystals. Therefore, amorphous materials in which still efficient (but less optimal than in crystals) $\pi$-stacking between the polymer chains is present, might show a similarly strong spin coupling, since a very poor $\pi$-interaction at the boundaries is replaced by a relatively effective $\pi$-interaction. This situation is somewhat reminiscent of the effect of crystallinity on the charge carrier mobilities in conjugated polymers ${ }^{24}$. Therefore, we hypothesize that the presence and strength of the ferromagnetism in the studied poly(thiophene)s is correlated with $\pi$-interactions between the polymer chains. Rather than the ESR spin density, the supramolecular organization seems to play a dominant role. Therefore, the difference in strength of the magnetic behavior of the poly(thiophene)s can be attributed to difference in the supramolecular organization of the different polymers. Indeed, the poly(thiophene)s with the worst interchain interactions (GRIM-P3AOT and, to a lesser extent, HTP3AOT, as is for instance visualized by DSC (see above)) also shows the lowest coercivity. This can also be correlated with the presence of a sharp, red-shifted absorption band in the UV-Vis spectrum of the polymers (see Supporting Information for the UV-vis spectra in a poor solvent mixture). Such band, with corresponding monosignate Cotton effect, has been attributed to a delocalized transition of many, stacked polymer chains and is therefore very sensitive to $\pi$-interactions. ${ }^{12,25}$ This band is the most strongly pronounced in HT-P3ATT, very clearly present in HH-TT-P3AOT and HT-P3AT, slightly visible HT-P3AOT and hardly detectable in GRIM-P3AOT. 


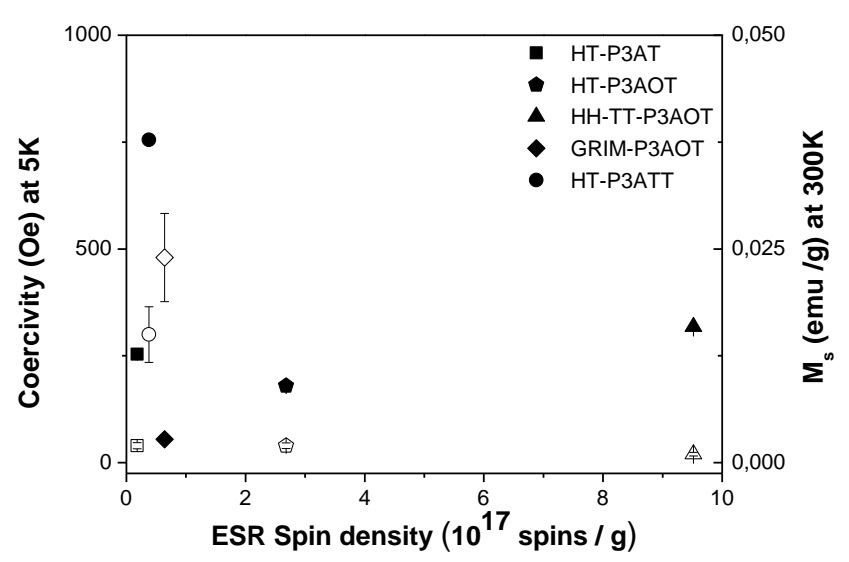

Figure 4. Correlation between the ESR spin density and the magnetic hysteresis width at $5 \mathrm{~K}$ (filled symbols) and the magnetization at saturation $\mathrm{M}_{\mathrm{s}}$ at $300 \mathrm{~K}$ (unfilled).

In order to further verify this hypothesis, the powders were partially oxidized by exposure to air, which increased the ESR spin density (Table 1). The supramolecular organization, however, can expected to be only slightly affected by this very moderate oxidation. As can be derived from Figure S6, the superpara- and ferromagnetic behavior is not influenced by oxidation due to contact with ambient air, which again clearly demonstrates than the ESR spin density is not the determining factor in the SQUID magnetic behavior of the poly(thiophene)s.

\section{Conclusion}

In conclusion, we have demonstrated that neutral substituted poly(thiophene)s show significant spin densities, which are governed by the nature of the substituent. Their presence is an intrinsic feature of the polymers. The distance between the spins is too large to allow spin coupling and therefore, these non-interacting spins give rise to a typical Curie-Weiss paramagnetic behavior. SQUID magnetometry reveals a superparamagnetic behavior at $300 \mathrm{~K}$ and ferromagnetism at $5 \mathrm{~K}$. This magnetic behavior does not originate from the ESR-active spin system and is therefore independent of the intensity of the ESR signal. The interaction between the magnetic moments seem to be very dependent on the supramolecular behavior of the conjugated polymers. 
Acknowledgment: We are grateful to the Research Fund of the Katholieke Universiteit Leuven, the Fund for Scientific Research (FWO-Vlaanderen), the Flemish Concerted Action (GOA), the Belgian Interuniversity Poles of Attraction (IAP), and the Air Force Office of Scientific Research. S. C. and J.C. are doctoral fellows of the Institute for the Promotion of Innovation through Science and Technology in Flanders (IWT-Vlaanderen) and G. K. is a postdoctoral fellow of the Fund for Scientific Research (FWO-Vlaanderen). We are also grateful to G. Absillis for performing the AAS measurements.

Supporting Information Available: ESR spectra of the polymers, SQUID measurements after aging and UV-vis spectra in a poor solvent mixture. This material is available free of charge via the Internet at http://pubs.acs.org.

\section{References}

(1) Skotheim, T. A., Elsenbaumer, R. L., Reynolds, J. R., Eds. Handbook of Conducting Polymers; Marcel Dekker, Inc.: New York, 1998.

(2) (a) Rajca, S.; Rajca, A.; Wongsriratanakul, J.; Butler, P.; Choi, S. J. Am. Chem. Soc. 2004, 126, 6972-6986. (b) Rajca, A.; Wongsriratanakul, J.; Rajca, S. J. Am. Chem. Soc. 2004, 126, 6608-6626. (c) Van Meurs, P. J.; Janssen, R. A. J. J. Org. Chem. 2000, 65, 5712-5719. (d) Fukuzaki, E.; Nishide, H. J. Am. Chem. Soc. 2006, 128, 996-1001. (e) Murata, H.; Daisuke, M.; Nishide, H. Macromolecules 2006, 39, 6331-6335. (f) Galecka, M.; Wielgus, I.; Zagorska, M.; Pawlowski, M.; Kulszewicz-Bajer, I. Macromolecules 2007, 40, 4924-4932.

(3) (a) Zuev, P. S.; Sheridan, R. S. Tetrahedron 1995, 51, 11337-11376. (b) Tomioka, H.; Hattori, M.; Hirai, K.; Sato, K.; Shiomi, D.; Takui, T.; Itoh, K. J. Am. Chem. Soc. 1998, 120, 1106-1107. (c) Hirai, K.; Kamiya, E.; Itoh, T.; Tomioka, H. Org. Lett. 2006, 8, 1847-1850.

(4) (a) Wasserman, E.; Murray, R. W.; Yager, W.A.; Trozzolo, A. M.;Smolynsky, G. J. Am. Chem. Soc. 1967, 89, 5067. (b) Mataga, N. Theor. Chem. Acta 1968, 10, 272. (c) Teki, Y.; Takui, T.; Itoh, K.; 
Iwamura, H.; Kobayashi, K. J. Am. Chem. Soc. 1986, 108, 2147. (d) Ovchinnikov, A. A. Dok. Nauk. Acad. USSR 1979, 236, 957. (e) Korshak, Y.; Medvedeva, T. V.; Ovhinnikov, A. A.; Spektor, V. N. Nature 1987, 325, 3701. (f) Lahti, P. M., Ed. Magnetic Properties of Organic Materials; Marcel Dekker, Inc.: New York, 1999.

(5) (a) Brédas, J. L.; Street, G. B. Acc. Chem. Res. 1985, 18, 309-315. (b) Su, W. P.; Schrieffer, J. R.; Heeger, A. J. Phys. Rev. Lett. 1979, 42, 1698-1701. (c) Fisher, A. J.; Hayes, W.; Wallace, D. S. J. Phys. Condens. Matter 1989, 1, 5567-5593.

(6) (a) Long, Y.; Chen, Z.; Shen, J.; Zhang, Z.; Zhang, L.; Xiao, H.; Wan, M.; Duvail, J.-L. J. Phys. Chem. B 2006, 110, 23228-23233. (b) Zaidi, N. A.; Giblin, S. R.; Terry, I.; Monkman, A. P. Polymer 2004, 45, 5683-5689. (c) Dallas, P.; Stamopoulos, D.; Boukos, N.; Tzitzios, V.; Niarchos, D.; Petridis, D. Polymer 2007, 48, 3162-3169. (d) Kahol, P. K.; Raghunathan, A.; McCormick, D. J. Synth. Metals 2004, 140, 261-267.

(7) Mizoguchi, K.; Kachi, N.; Sakamoto, H.; Kume, K.; Yoshioka, K.; Masubuchi, S.; Kazama, S. Synth. Metals 1997, 84, 695-698.

(8) (a) Nascimento, O. R.; de Oliveira, A. J. A.; Correa, A. A.; Bulhões, L. O. S.; Pereira, E. C. Phys. Rev. $B$ 2003, 67, 144422. (b) Nascimento, O. R.; de Oliveira, A. J. A.; Pereira, E. C.; Correa, A. A.; Walmsley, L. J. Phys. Condens. Matter 2008, 20, 035214. (c) de Paula, F. R.; Walmsley, L.; Pereira, E. C.; de Oliveira, A. J. A. J. Magn. Magn. Materials 2008, 320, 193-195. (d) Sugiyama, K.; Kojima, T.; Fukuda, H.; Yashiro, H.; Matsuura, T.; Shimoyama, Y. Thin Solid Films 2008, 516, 2691-2694 (e) Nalwa, H. S. Phys. Rev. B 1989, 39, 5964-5974. (e) Konkin, A.; Roth, H.-K.; Scharff, P.; Aganov, A.; Ambacher, O.; Sensfuss, S. Solid State Commun. 2009, 149, 893-897.

(9) (a) Nechtschein, M.; Devreux, F.; Genoud, F.; Gugliemi, M.; Holczer, K. Phys. Rev. B 1983, 27, 61-78. 
(10) (a) McCullough, R. D.; Lowe, R. L.; Jayaraman, M.; Anderson, D. L. J. Org. Chem. 1993, 58, 904-912. (b) Lowe, R. S.; Khersonsky, S. M.; McCullough, R. D. Adv. Mater. 1999, 11, 250-253. (c) Bidan, G.; Guillerez, S.; Sorokin, V. Adv. Mater. 1996, 8, 157-160.

(11) Koeckelberghs, G.; Vangheluwe, M.; Samyn, C.; Persoons, A.; Verbiest, T. Macromolecules 2005, 38, 5554-5559.

(12) Vandeleene, S.; Van den Bergh, K.; Verbiest, T.; Koeckelberghs, G. Macromolecules, 2008, 41, 5123-5131.

(13) Vangheluwe, M.; Verbiest, T.; Koeckelberghs, G. Macromolecules 2008, 41, 1041-1044.

(14) Stesmans, A. Phys. Rev. B 1993, 48, 2418- 2435.

(15) Miyakoshi, R.; Yokoyama, A.; Yokozawa, T. J. Am. Chem. Soc. 2005, 127, 17542-17547.

(16) (a) Kanemoto, K.; Kato, T. Phys. Rev. B 2003, 68, 092302. (b) Kanemoto, K.; Furukawa, K.; Negishi, N.; Aso, Y.; Otsubo, T. Phys. Rev. B 2007, 76, 155205.

(17) (a) Krinichnyi, V. I. Synth. Met. 2000, 108, 173-222. (b) Mizoguchi, K.; Honda, M.; Kachi, N.; Shimizu, F.; Sakamoto, H.; Kume, K. Solid State Commun. 1995, 96, 333-337. (c) Harima, Y.; Eguchi, T.; Yamashita, K.; Kojima, K.; Shiotani, M. Synth. Metals 1999, 105, 121-128.

(18) Elliott, T. J. Phys. Rev. 1954, 96, 266-279.

(19) (a) Houzé, E.; Nechtschein, M. Phys. Rev. B 1996, 53, 14309-14318. (b) Nechtschein, M.; Genoud, F. Solid State Commun. 1994, 91, 471. (c) Bartl, A.; Dunsch, L.; Naarmann, H.; Schmeisser, D.; Gopel, W. Synth. Met. 1993, 61, 167. (d) Inoue, M.; Inoue, M. B.; Castillo-Ortega, M. M.; Mizuno, M.; Asaji, T.; Nakamura, D. Synth. Met. 1989, 33, 355. 
(20) Yamamoto, T.; Muramatsu, Y.; Shimizu, T.; Yamada, W. Macromol. Rapid Commun. 1998, 19, 263-266.

(21) Abdou, M. S. A.; Orfino, F. P.; Son, Y.; Holdcroft, S. J. Am. Chem. Soc. 1997, 119, 4518-4524.

(22) (a) Mizes, H. A.; Conwell, E. M. Phys. Rev. Lett. 1993, 70, 1505-1508. (b) Kuroda, S.; Noguchi, T.; Ohnishi, T. Phys. Rev. Lett. 1994, 72, 286-289.

(23) Nishide, H.; Iwasa, S.; Pu, Y.-J.; Suga, T.; Nakahara, K.; Satoh, M. Electrochim. Acta 2004, 50, $827-831$.

(24) Liu, J.; Zhang, R.; Sauvé, G.; Kowalewski, T.; McCullough, R. D. J. Am. Chem. Soc. 2008, 130, 13167-13176.

(25) Crispin, X.; Cornil J.; Friedlein R.; Okudaira K. K.; Lemaur V.; Crispin A.; Kestemont G.; Lehmann M.; Fahlman M.; Lazzaroni R.; Geerts Y.; Wendin G.; Ueno N.; Bredas J.-L.; Salaneck W. R J. Am. Chem. Soc. 2004, 126, 11889-11899. 


\section{For Table of Contents use only}

Magnetic Properties of Substituted Poly(thiophene)s in Their Neutral State.

Steven Vandeleene, Mihaela Jivanescu, André Stesmans, Jo Cuppens, Margriet J. Van Bael, Hitoshi Yamada, Norio Sato, Thierry Verbiest, and Guy Koeckelberghs*

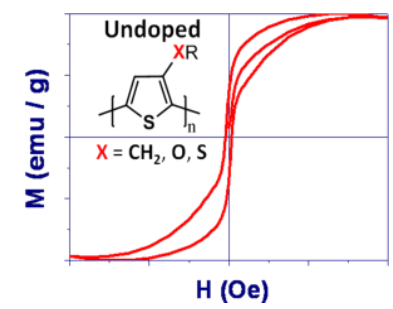

